\title{
Análisis de la Socialización sobre Perfiles de la dinámica bullying"
}

Analysis of Socialization on Bullying Dynamics Profiles

Recibido: 21 de noviembre de 2013 | Revisado: 25 de junio de 2014 | Aceptado: 17 de enero de 2015

doi:10.11144/Javeriana.upsy14-3.aspd

Para citar este artículo: Polo del Río, M. I., León del Barco, B., Felipe, E., Fajardo, F., \& Gómez, T. (2015). Análisis de la Socialización sobre Perfiles de la dinámica bullying. Universitas Psychologica, 14(3), 1117-1128. http://dx.doi.org/10.11144/Javeriana. upsy14-3.aspd

* $\quad$ Artículo de investigación. Título del Proyecto: "Análisis de la Convivencia Escolar en centros educativos de la Comunidad Extremeña". Entidad Financiadora: Consejería de Economía, Comercio e Innovación. Junta de Extremadura. Fecha de realización: Desde 18/01/2008-Hasta 18/08/2011. Código de registro: PRI08A035.

** Profesora Asociada y Doctora en Psicología. Departamento de Psicología y Antropología. Facultad de Formación del Profesorado. Miembro del grupo de Investigación en Psicología Educativay Social (GIPES). Universidad de Extremadura. Campus Universitario, avda. Universidad s/n. 10070 Cáceres. Tel. 927257049 (Ext.51273).Correo electrónico:mabelpdrio@unex.es

***: Profesor Titular de Psicología de la Educación. Facultad de Formación del Profesorado. Coordinador del Grupo de Investigación en Psicología Educativa y Social (GIPES). Universidad de Extremadura. Miembro colaborador del Centro de Investigaçao en Educaçao e Psicologia delaUniversidaddeÉvora.Correo electrónico: bleon@ unex.es

***:*at Profesora Titular de Personalidad, Evaluación y Tratamiento Psicológico. Facultad de Formación del Profesorado. Miembro del Grupo de Investigación en Psicología Educativa y Social (GIPES). Universidad de Extremadura.Correo electrónico: efelipe@unex.es

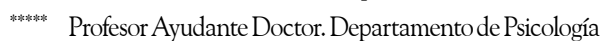
y Antropología. Facultad de Formación del Profesorado. Miembrodel Grupo de Investigación en Psicología Educativa y Social (GIPES). Universidad de Extremadura. Correo electrónico: fernadofajardo@unex.es

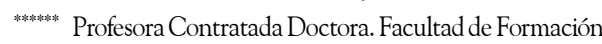
del Profesorado. Miembro del Grupo de Investigación en Psicología Educativa y Social (GIPES). Universidad de Extremadura. Miembro colaborador del Centro de Investigaçao en Educaçao e Psicologia de la Universidad de Évora.Correo electrónico: tgomez@unex.es

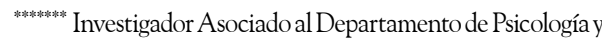
Antropología. Miembro del Grupo de Investigación en Psicología Educativa y Social (GIPES). Universidad de Extremadura. Correo electrónico: smendo@unex.es

\author{
MARÍA ISABel Polo DEL RÍO** \\ BENITO LEÓN DEL BARCO *** \\ ELENA FeliPE CASTAÑO $* * * *$ \\ FERNANDO FAJARDO BULLÓN ***** \\ TERESA GÓMEZ CARROZA ******* \\ Universidad de Extremadura, Cáceres, España \\ SANTIAGO MENDO LÁZARO ********
}

\section{RESUMEN}

Con esta investigación, mediante un análisis discriminante se pretende analizar cuáles variables de socialización presentan un mayor poder de discriminación o cuantifican mejor las diferencias entre los distintos niveles de agresión y victimización en el fenómeno bullying. La muestra está formada por 700 adolescentes estudiantes. Se utilizó el Cuestionario de Convivencia Escolar (Defensor del Pueblo, 2006) y el BAS-3 (Batería de Socialización, en formato de autoevaluación) de Silva y Martorell (1989). Nuestros resultados confirman que niveles altos de agresión en el acoso escolar estarían caracterizados por puntuaciones bajas en consideración hacia los demás y autocontrol en las relaciones sociales, mientras que niveles altos de victimización estarían caracterizados por puntuaciones altas en ansiedad social/ timidez y retraimiento social.

Palabras clave

socialización; bullying; adolescentes; agresores; víctimas

\section{A B S T R A C T}

With this research, by discriminant analysis to analyze variables of socialization that have a higher discriminatory or better quantify the differences between the different levels of aggression and victimization in the bullying phenomenon. The sample consisted of 700 adolescent students. Questionnaire was used School Coexistence (Ombudsman, 2006) and the BAS-3 (Drums of socialization, self-assessment format) de Silva and Martorell (1989). Our results confirm that high levels of aggression in bullying would be characterized by low scores on consideration for others and self in social relations, while high levels of victimization, would be characterized by high scores on social anxiety / shyness and social withdrawal.

Keywords

socialization; bullying; adolescence; bullies; victims 


\section{Introducción}

El acoso escolar es una forma de agresión especialmente dañina y perjudicial (Trianes, Muñoz, \& Jiménez, 2007) que afecta muy seriamente al bienestar psicológico y social de quien lo sufre (Buelga, Cava, \& Musitu, 2012a, 2012b), y es definido como una "conducta de persecución física y/o psicológica que realiza un/a alumno/a contra otro/a, al que escoge como víctima de repetidos ataques" (Olweus, 1983 p. 353).

La preocupación social que suscita la violencia en los contextos sociales ha dado lugar a numerosas intervenciones y acciones en diferentes países y en muy diferentes ámbitos (Martín, Fernández, Andrés, Del Barrio, \& Echeita, 2003). La mayoría de las investigaciones se centran en el ámbito escolar y pretenden dos objetivos: en primer lugar, la investigación epidemiológica de la gravedad del fenómeno en cada contexto o entorno cultural (país), mediante cuestionarios u otros procedimientos de recogida de información, y en segundo lugar, una labor de intervención dirigida hacia todos los elementos implicados, individuales (agresor y víctima), familiares, escolares (organización y políticas educativas) y sociales (marco legal y políticas sociales), tanto para detener el proceso como para su prevención. Para Garaigordobil y Oñederra, (2010a, 2010b) en los últimos años se ha pasado de no intervenir a realizar cantidad de estudios y campañas preventivas en los centros.

Un área de estudio importante en el acoso escolar continúa siendo el análisis de las causas del fenómeno y de los factores que actuarían como protección/riesgo en los ámbitos culturales, sociales, personales, escolares y familiares. La socialización puede ser clave como factor de protección o de riesgo en la aparición de situaciones de violencia escolar (Baldry \& Farrington, 2005). La infancia y la adolescencia son periodos de la vida en los que la socialización primaria y las primeras etapas de la socialización secundaria son momentos especialmente importantes para su conformación social (Lahire, 2007).

En la actualidad, niños y adolescentes están sometidos a múltiples agentes socializadores muy significativos para ellos: familia, grupo de iguales, institución escolar y otros entornos sociales. En concreto, las experiencias con los iguales suponen un vehículo de aprendizaje de habilidades y actitudes que influyen en la adaptación social de la persona a lo largo de toda su vida, y que determinan su bienestar y ajuste social, emocional y cognitivo (Musitu \& Cava, 2003). Pero también pueden constituir el terreno perfecto para abonar expresiones de malestar emocional y comportamientos violentos (Musitu, Jiménez, \& Povedano, 2009) y aparecer ciertos conflictos, uno de los cuales, el acoso escolar o maltrato entre iguales, genera una importante fuente de dificultades y frustraciones para el alumnado involucrado (Martínez-Antón, Buelga, \& Cava, 2007), y va más allá de los episodios concretos de agresión y victimización, llegando a constituir una dinámica asidua de exclusión, violencia y deterioro de la socialización (Cerezo, 2009).

El acoso escolar produce una perversión de las relaciones entre agresor y víctima, al desaparecer la relación de igualdad y ser sustituida por una relación jerárquica de dominación-sumisión (Defensor del Pueblo, 1999). Según Cerezo (2002), los agresores presentan un perfil específico frente a las víctimas, resultando relevante su fortaleza física, la provocación y su carácter de cierto liderazgo frente a la cobardía, ansiedad y sentimientos de antipatía que despiertan las víctimas, lo que se puede interpretar como elemento favorecedor de bullying en el ámbito escolar.

El agresor, alumno que maltrata a la víctima, lo puede hacer de dos formas: bien iniciando el acoso por cuenta propia, es el agresor activo (Perren \& Alsaker, 2006) que goza de popularidad entre sus compañeros, o bien, el agresor pasivo que no inicia el maltrato, pero apoya al agresor activo (Olweus, 1993). Estudios realizados hasta ahora coinciden en que el alumno agresor presenta un estilo de socialización concreto, tiene dificultad para ponerse en el lugar de los otros (Olweus, 1993), presenta deficiencias en autocontrol en las relaciones sociales (Avilés \& Monjas, 2005; Díaz-Aguado, 2005; Cerezo, 2008; Caspi et al., 1994), muestra indiferencia (Caurcel \& Almeida, 2008) y con el tiempo se acostumbra a vivir abusando de los demás, lo que le impide inte- 
grarse de forma adecuada a la vida social del centro educativo (Benítez \& Justicia, 2006).

Por otro lado, se encuentra la víctima, sujeto que recibe las agresiones de otro de manera sistemática, generalizando la percepción hostil al conjunto del ambiente escolar, y generando, entre otros, graves estados de ansiedad o depresivos (León, 2009; Perren \& Alsaker, 2006) y aislamiento social (Cerezo, 2002; Rigby, 2000), destrucción de la autoestima y confianza en sí mismo, difícil adaptación social y, en situaciones extremas, el suicidio. Martínez-Monteagudo, Inglés, Trianes y García-Fernández (2011) revelan una casuística que afecta al alumnado con problemas de ansiedad, el haber estado implicado en agresiones como víctima.

Diversos estudios (Díaz-Aguado, Martínez, \& Martín, 2004; Olweus, 1993; Pellegrini, Bartini, \& Brooks, 1999; Salmivalli, Lagerspetz, Björkqvist, Österman, \& Kauklainen, 1996; Schwartz, Dodge, Pettit, \& Bates, 1997; Smith, Talamelli, Cowie, Naylor, \& Chauhan, 2004) muestran dos perfiles de víctima, con estilos de socialización característicos. La víctima pasiva se caracteriza por baja autoestima, ansiedad y depresión, tener pocos amigos y sufrir consecuencias adversas, como ser aislada socialmente por sus compañeros (Benítez \& Justicia, 2006). Y la víctima activa, también aislada socialmente, pero con un matiz distinto en cuanto a sus relaciones sociales con el resto de compañeros: ser poco popular e incluso rechazada por ellos.

Con esta investigación, mediante un análisis discriminante, se pretende analizar cuáles variables de la socialización presentan un mayor poder de discriminación entre los diferentes niveles de intensidad de agresión y de victimización.

\section{Método}

\section{Participantes}

La muestra total estuvo formada por 700 adolescentes estudiantes, $43 \%$ mujeres y $57 \%$ varones con una media de edad de $13.98(\mathrm{DE}=1.38+)$. El número de participantes se determinó a partir del número de alumnos matriculados en Educación Secundaria Obligatoria (ESO) en centros públicos y concertados de la Comunidad de Extremadura, durante el curso 2010-2011, considerando un error muestral de $3 \%$ y un nivel de confianza de $95.5 \%$. La selección de la muestra se realizó mediante un muestreo polietápico por conglomerados y selección aleatoria de los grupos, en los centros que disponían

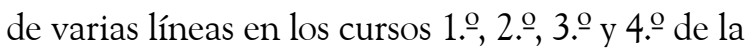
ESO. El muestreo por conglomerados se llevó a cabo seleccionando al azar cuatro centros. En cuanto a la distribución por curso de nuestros participantes, 190 alumnos eran de 1. de ESO, 177 de 2. de ESO, 171 de 3. y 162 de 4. de ESO.

\section{Instrumentos}

\section{Cuestionario de Convivencia Escolar (Defensor del Pueblo, 2007)}

Se utilizaron los resultados de tres ítems en los que se pedía que contestaran según una escala de tipo Likert de cuatro intervalos, que van de 1 nunca a 4 siempre, si habían vivido las 13 situaciones de acoso escolar desde la perspectiva de víctima, agresor/a y observador/a. Las situaciones son las siguientes: ignorarle, no dejarle participar, insultarle, ponerle motes que le ofenden o ridiculizan, hablar mal de él o ella, esconderle cosas, romperle cosas, robarle, pegarle, amenazarle para meterle miedo, acosarle sexualmente, obligarle con intimidaciones a hacer cosas que no quiere hacer y amenazarle con armas.

\section{El BAS-3 (Batería de socialización, en formato de autoevaluación) de Silva y Martorell (1989)}

Se compone de 5 escalas de socialización: 1 . Consideración hacia los demás, con 14 elementos, detecta sensibilidad social o preocupación por los demás, en particular por aquellos que tienen problemas y son rechazados. 2. Autocontrol en las relaciones sociales se compone de 14 elementos y recoge una dimensión que representa en su polo positivo, acatamiento de normas y reglas sociales que facilitan la convivencia y el respeto, y en el polo negativo, conductas agresivas. 3. Retraimiento social, con 14 elementos, detecta aislamiento de los demás. 4. Ansiedad social/timidez, con 12 elementos, en 
los cuales se detecta manifestaciones de ansiedad unidas a reacciones de timidez. 5. Liderazgo, con 12 elementos, se detecta popularidad, confianza en sí mismo y espíritu de servicio.

En relación con la consistencia interna, se encuentra en límites muy satisfactorios (Silva \& Martorell, 1989); después de someter los datos a análisis factoriales, se llegó a una estructura final de 65 elementos y 5 escalas que se construyeron con los elementos de más alta saturación, escalas que se han descrito anteriormente. La estabilidad temporal, según los autores, es asimismo satisfactoria, excepto para las escalas Consideración con los demás y Retraimiento social, las cuales deben tomarse con cautela para estudios longitudinales.

\section{Procedimiento}

Los datos que se presentan forman parte de una investigación más amplia relacionada con la convivencia escolar y bullying, en centros de educación primaria y secundaria. Uno de los objetivos fue conocer y analizar la relación entre conductas de agresión y victimización con variables de socialización.

El procedimiento seguido para la obtención de datos fue la administración de los cuestionarios por grupo de clase. Previamente, se había informado de los objetivos de la investigación al Centro y a los padres y se había solicitado su permiso para la administración y a los participantes, el consentimiento informado de forma verbal. Se les aseguró la confidencialidad de los datos obtenidos y su utilización exclusiva para fines de investigación. La administración de los cuestionarios tomó cerca de una hora y se llevó a cabo a lo largo de dos meses, durante el tiempo de tutorías.

\section{Resultados}

En primer lugar se seleccionaron diferentes subgrupos de la muestra final en función de los roles asumidos por los participantes. Para ello, se utilizaron las puntuaciones en los tres ítems del Cuestionario del Defensor del Pueblo (2007), en los que se preguntaba a los sujetos por su participación, según los tres roles descritos por Olweus (1993), en diversas situaciones de bullying. En total son 13 situaciones distintas que se han enumerado en el apartado de instrumentos y a las que los sujetos debían responder en función de si habían sufrido (víctima), observado (observador) o provocado (agresor) esa situación. Para calcular la frecuencia de participación en ese suceso, se realizó un sumatorio de las respuestas a esos 13 ítems, de manera que se obtuvieron dos nuevas variables, denominadas intensidad de la experimentación de la situación de víctima y agresor. En estas variables, se obtuvieron valores de entre 13 y 52, indicando el valor de 13: ausencia de experiencias y el valor 52: gran presencia de experiencias en todas las situaciones de bullying.

Una vez obtenidas estas variables, se calcularon: el valor de percentil 25 para seleccionar aquellos participantes que hubiesen experimentado un menor número e intensidad de las situaciones y el valor de percentil 75 para aquellos que hubiesen experimentado un mayor número e intensidad de las situaciones. Las puntuaciones descriptivas de la variable víctima y los valores de los percentiles para cada uno de los niveles de intensidad fueron los siguientes: (M $=14.53, \mathrm{DE}=2.55$, percentil $25=13$, percentil 75 $=15)$, para la variable agresor: $(M=15.65, D E=$ 3.97, percentil $25=13$, percentil $75=16$ ).

El análisis discriminante permite determinar la existencia de diferencias significativas entre los diferentes niveles de intensidad de victimización en el bullying en relación con el conjunto de variables de Socialización, por un lado, y la existencia de diferencias significativas entre los diferentes niveles de intensidad de agresión en el bullying en relación con el conjunto de variables de Socialización, por otro. Igualmente, mediante el análisis discriminante, se pretendió clasificar a los participantes y asignarlos a cada uno de los niveles de intensidad de victimización y agresión en el bullying, respectivamente, en función del resultado de la combinación lineal del conjunto de variables independientes. En el presente estudio, se utilizaron como variables independientes y predictoras las cinco escalas de Socialización del BAS-3 y como variables dependientes, los diferentes niveles de intensidad de victimización y agresión en el bullying. 
La Tabla 1 muestra las medias y desviaciones estándar de cada uno de los niveles de intensidad de victimización y de agresión en la dinámica bullying, para las cinco escalas de Socialización. En relación con los diferentes niveles de victimización, podemos observar, que niveles altos de victimización presentan puntuaciones más altas en Consideración hacia los demás y más bajas en Autocontrol en las relaciones sociales que los niveles más bajos. Y que a medida que aumenta el nivel de victimización, aumenta el Retraimiento Social y la Ansiedad Social/Timidez.

Con respecto a los diferentes niveles de agresión del bullying, vale destacar que niveles altos de agresión presentan puntuaciones más bajas en Consideración hacia los demás y en Autocontrol en las relaciones sociales.

A continuación, se examinó la posible existencia de diferencias entre las medias de los tres grupos de niveles de victimización y entre las medias de los tres grupos de niveles de agresión en el bullying, en cuanto a las puntuaciones en las escalas de Socialización del BAS-3. Para ello, se realizó un análisis de la varianza (ANOVA), encontrando diferencias significativas entre los tres niveles de victimización para dos de las escalas de Socialización del BAS3: la escala Autocontrol en las relaciones sociales (Wilks $\lambda=0.988, F=5.343, p<0.048$ ); Ansiedad Social/Timidez (Wilks $\lambda=0.979, F=5.343, p<$ 0.005). Del mismo modo, se encontraron diferencias significativas entre los tres niveles de agresión para tres de las escalas de Socialización del BAS-3: Consideración hacia los demás (Wilks $\lambda=0.902$, $F=26.864 p=0$ ), Autocontrol en las relaciones sociales (Wilks $\lambda=0.906, F=25.4015 p=0$ ) y Retraimiento Social (Wilks $\lambda=0.983, F=4.379$ $p=0.013)$.

Una vez demostrada la existencia de diferencias entre la medias de los tres niveles de victimización y la existencia de diferencias entre la medias de los tres niveles de agresión del bullying, mediante el análisis discriminante, se determinó cuáles escalas de Socialización del BAS-3 explican en mayor medida esas diferencias. En la Tabla 2 se muestra la matriz de estructura que se crea en el análisis discriminante, correspondiente a victimización y a agresión. El número máximo de funciones discriminantes o combinaciones lineales es igual a una unidad menos que el número de grupos asignados a la variable dependiente. ¿Qué función tiene un mayor poder de discriminación y se utilizará para interpretar los datos? El análisis de las funciones discriminantes indica que la Función 1 es la que presenta un mayor poder de discriminación entre los tres niveles de victimización y los de agresión.

La Función 1 explica un porcentaje de varianza muy superior al resto de funciones, muestra una mayor correlación canónica y distancia entre los grupos discriminados (lambda de Wilks más cercano a 0). Además, el análisis de chi cuadrado presenta el más elevado nivel de significación. Para la victimización, Función 1 (\% de varianza

\section{TABLA 1}

Medias y desviaciones típicas Escalas de Socialización del BAS-3 en función de los diferentes niveles de victimización y agresión del bullying

\begin{tabular}{lccccc}
\hline \multicolumn{5}{c}{ Escala de Socialización BAS-3 } \\
\hline & $\begin{array}{c}\text { Consideración } \\
\text { hacia los demás } \\
\text { M(DE) }\end{array}$ & $\begin{array}{c}\text { Autocontrol en las } \\
\text { relaciones sociales } \\
\text { M(DE) }\end{array}$ & $\begin{array}{c}\text { Retraimiento So- } \\
\text { cial M(DE) }\end{array}$ & $\begin{array}{c}\text { Ansiedad Social/ } \\
\text { Timidez M(DE) }\end{array}$ & Liderazgo M(DE) \\
\cline { 2 - 5 } Baja Victimización & $25.62(3.25)$ & $24.72(2.95)$ & $15.65(3.39)$ & $15.33(3.29)$ & $18.32(2.51)$ \\
Media Victimización & $26.19(3.14)$ & $24.77(4.33)$ & $16.02(3.32)$ & $16.04(2.74)$ & $18.43(2.98)$ \\
Alta Victimización & $26.33(2.78)$ & $23.75(3.74)$ & $16.52(2.63)$ & $16.47(3.25)$ & $18.30(2.55)$ \\
Baja Agresión & $26.578(3.2)$ & $25.507(2.75)$ & $16.407(4.51)$ & $16.164(3.83)$ & $18.542(3.04)$ \\
Media Agresión & $26.524(2.69)$ & $24.991(3.71)$ & $15.510(2.5)$ & $15.882(2.86)$ & $18.37(2.59)$ \\
Alta Agresión & $24.278(3.26)$ & $22.659(3.84)$ & $16.317(2.54)$ & $15.516(2.97)$ & $18.008(2.43)$ \\
\hline
\end{tabular}

Fuente: elaboración propia 
María Isabel Polo del Río, Benito León del Barco,

Elena Felipe Castaño, Fernando Fajardo Bullón, Teresa Gómez Carroza

TABLA 2

Matriz de la estructura. Variables ordenadas por el tamaño de la correlación con la función discriminante

\begin{tabular}{lcccc}
\hline & \multicolumn{2}{c}{ Funciones(victimización) } & \multicolumn{2}{c}{ Funciones (agresión) } \\
\hline & Función 1 & Función 2 & Función 1 & Función 2 \\
Ansiedad Social/Timidez & $0.616^{*}$ & 0.367 & 0.157 & 0.235 \\
Retraimiento Social & $0.441^{*}$ & -0.062 & -0.117 & 0.964 \\
Autocontrol en las relaciones sociales & -0.386 & 0.882 & $0.738^{*}$ & 0.221 \\
Consideración hacia los demás & 0.395 & 0.503 & $0.759^{*}$ & 0.213 \\
Liderazgo & -0.002 & 284 & 0.167 & 0.145 \\
\hline
\end{tabular}

Fuente: elaboración propia

$=91.3$, correlación canónica $=0.229$, Wilks $\lambda$ : $\left.0.942, \chi^{2}=29.026, g l=10, p<0.001\right)$. Por tanto, según la Función 1 el factor que mayor capacidad discriminante tiene entre los niveles de victimización es Ansiedad Social/Timidez (0.616). Le sigue el factor Retraimiento Social (0.441). Para poder interpretar la relación de cada factor con los diferentes niveles, se requiere conocer las medias y especialmente el signo de las funciones en los centroides de los grupos. Para el grupo Baja Victimización $=-0.232$, para el grupo Media Victimización $=0.052$ y para el grupo Alta Victimización $=0.38$. Especialmente, el grupo de Alta Victimización se caracterizaría por tener una mayor Ansiedad y Retraimiento Social.

Para la agresión, Función 1 (\% de varianza $=$ 92, correlación canónica $=0.398$, Wilks $\lambda$ : 0.828, $\chi^{2}=92.523, g l=10, p=0$ ), los factores que mejor cuantifican las diferencias entre los diferentes niveles de agresión son Autocontrol en la Relaciones Sociales $(0.738)$ y Consideración hacia los demás (0.759). Las medias y el signo de las funciones en los centroides de los grupos han sido: grupo Baja Agresión $=0.319$, Media Agresión $=-0.21$ y para el grupo Alta Agresión = -0.736. Este último grupo, se caracteriza por tener poco Autocontrol y Consideración hacia los demás.

Finalmente, en la Tabla 3 se observa que la función canónica discriminante obtenida permite clasificar correctamente el $57.7 \%$ del nivel baja intensidad de victimización, el $23.4 \%$ del nivel media intensidad de victimización y el 50.9\% del nivel alta intensidad de victimización. Tanto para el grupo de baja victimización como para el grupo de alta victimización se obtienen ganancias medias en las predicciones superiores al $33 \%$, que se acertaría por azar en los 3 niveles de intensidad de victimización. La Ansiedad y el Retraimiento Social ayudarían a discriminar ambos grupos.

En la Tabla 4 se aprecian ganancias medias en las predicciones superiores al $33 \%$, que se acertarían por azar en los 3 niveles de intensidad de agresividad, para el grupo de media intensidad de agresión $(53.3 \%)$ y para el grupo de alta intensidad de agresión (62.7\%). La falta de Autocontrol y Consideración hacia los demás sería una característica de los sujetos altamente agresores, principalmente.

TABLA 3

Resultados de la clasificación empleando la función discriminante

\begin{tabular}{ccccc}
\hline & & \multicolumn{3}{c}{ Grupo de pertenencia pronosticado } \\
\hline & & Baja victimización & Media victimización & Alta victimización \\
\hline$\%$ & Baja victimización & 57.7 & 19.2 & 23 \\
& Media victimización & 38.3 & 23.4 & 38.3 \\
Alta victimización & 33 & 16 & 50.9 \\
\hline
\end{tabular}

Clasificados correctamente el $44.1 \%$ de los casos agrupados originales

Fuente: elaboración propia 


\section{TABLA 4}

Resultados de la clasificación empleando la función discriminante

\begin{tabular}{ccccc}
\hline & & \multicolumn{3}{c}{ Grupo de pertenencia pronosticado } \\
\hline$\%$ & Baja agresión & Baja agresión & Media agresión & Alta agresión \\
\hline Media Agresión & 26.4 & 56.4 & 17.1 \\
& Alta Agresión & 23.6 & 53.3 & 23.1 \\
& 15.9 & 21.4 & 62.7 \\
\hline
\end{tabular}

Clasificados correctamente el $48.1 \%$ de los casos agrupados originales.

Fuente: elaboración propia

\section{Discusión}

En este estudio, se planteó como objetivo principal analizar cuáles variables de socialización presentan un mayor poder de discriminación o cuantifican mejor las diferencias entre los diferentes niveles de intensidad de agresión y de victimización en el bullying. Los dos factores que mejor pueden discriminar los grupos en cuanto a la agresión son el factor Consideración hacia los demás y Autocontrol en las relaciones sociales. Y los dos factores que mejor cuantifican las diferencias entre los diferentes niveles de intensidad de victimización son por este orden el factor Ansiedad Social/Timidez y el factor Retraimiento Social. Concretamente, el análisis para el perfil agresor confirma que niveles altos de agresión estarían caracterizados por puntuaciones bajas en Consideración hacia los demás y Autocontrol en las relaciones sociales, es decir, el estilo de socialización para el alumno con alto nivel de agresión estaría caracterizado por baja consideración hacia sus compañeros y bajo autocontrol en sus relaciones sociales. Como concluyen los estudios de Hunter, Mora-Merchán y Ortega (2004), McConville y Cornell (2003), Olweus y Endresen (1998), los agresores suelen ser alumnos que muestran impulsos socialmente dominantes y una actitud positiva hacia el uso de la violencia. Se coincide en este estudio con el perfil social del agresor que presentan Montañés, Bartolomé, Parra y Montañés (2009), en cuanto a la baja consideración hacia los demás y el bajo autocontrol en las relaciones sociales. Y con Cerezo (2002), cuya investigación llevada a cabo con una muestra de 46 alumnos de dos grupos de Tercero de Educación
Secundaria, utilizando el BAS-3 (Batería de socialización, en formato de autoevaluación) de Silva y Martorell (1989), concluye según sus datos que los valores de consideración hacia los demás son bajos en toda la muestra. Según este mismo autor, en un estudio realizado en 2009 que analiza la situación del bullying en las aulas españolas, se afirma que el clima afectivo generado en el aula sufre una importante pérdida de conductas prosociales entre el alumnado, favoreciendo la falta de consideración hacia los demás.

Y para el perfil víctima, los resultados del presente trabajo confirman que niveles altos de victimización en el acoso escolar estarían caracterizados por puntuaciones altas en Ansiedad Social/Timidez y Retraimiento Social. Esto es, a mayor nivel de agresión sufrido por la víctima, el estilo de socialización se caracterizaría tanto por alta ansiedad social y timidez, como por alto retraimiento social. Resultados coincidentes con las investigaciones realizadas por Cerezo (2001, 2002), Rigby (2000) y Buelga et al. (2012a, 2012b) que concluyen que la víctima del acoso escolar presenta altas tasas de ansiedad y timidez en sus relaciones, es la más rechazada y menos reconocida socialmente dentro del grupo, lo que provoca en ella sentimientos de soledad y aislamiento social (Cerezo \& Ato, 2005).

En este sentido, se plantea si ies el estilo de socialización que presenta la víctima lo que determina que sea acosada por el agresor, o bien, es un estilo de socialización generado, tras ser victimizada? Por un lado, según Buelga et al. (2012a, 2012b), distintos estudios muestran que adolescentes con ciertas características personales y sociales (baja popularidad y autoestima, altos niveles de ansiedad, 
con sentimientos de soledad y síntomas depresivos, y rechazo por parte de sus compañeros) tienen más probabilidad de ser victimizados por sus compañeros. Características que suponen un factor de riesgo para sufrir acoso escolar y que, junto a las consecuencias negativas que se generan, puede derivar en un ciclo retroactivo del que la víctima no va a saber salir (Cava, Musitu, \& Murgui, 2007), llegando a encontrarse en una clara situación de indefensión en el grupo.

Por otro lado, Moreno, Vacas y Roa (2006) señalan que es el agresor, cuya acción negativa e intencionada, dirigida a desprestigiar socialmente a la víctima (Benítez \& Justicia, 2006), quien provoca en ella un aislamiento, una introversión social que la aísla aún más de su entorno (Oñate \& Piñuel, 2005) y una progresiva exclusión social del mismo (Griffin \& Gross, 2004). En este sentido, estudios como los realizados por Cava, Buelga, Musitu y Murgui (2010) remarcan la importancia que tiene la violencia relacional o indirecta en el ajuste psicosocial de los adolescentes, de manera que ciertas situaciones de violencia física y verbal, sin coexistir al mismo tiempo una exclusión y rechazo social, no se relacionarían, al menos durante la etapa de la adolescencia, con dificultades de ajuste psicosocial. Las consecuencias del acoso escolar dejan huellas imborrables en las víctimas, tanto a medio como a corto plazo, que comienzan a percibir el entorno social como poco seguro y amenazante (Cerezo, 1997; Olweus, 1998; Sutton \& Smith, 1999).

Por todo ello, se cree necesaria la implementación de programas sobre la prevención del acoso escolar en todos los centros educativos, y en coincidencia con Díaz-Aguado (2005) se enfatiza la importancia de erradicar las situaciones de exclusión desde las primeras etapas educativas y de favorecer la identificación de los adolescentes con los valores de respeto, empatía y no violencia, así como la necesidad de mantener una perspectiva ecológica (Berger, 2012) y colaborativa entre las familias, centros educativos y recursos comunitarios (Avilés, 2009). En relación con esto, cabría indicar que autores como Avilés (2006), Del Barrio, Martín, Montero, Gutiérrez, Barrios y De Dios (2008), Defensor del Pueblo (2007), Díaz-Aguado (2005),
Silva y Martorell (1989) y Garaigordobil y Oñederra (2010) consideran el factor socialización, junto con la familia y el entorno, decisivos en el fenómeno bulling, aunque de momento las investigaciones sean escasas.

Los autores consideran interesante, entonces, introducir variables, como son los estilos parentales y otras variables familiares, que puedan también relacionarse con la implicación en la dinámica bullying. En este sentido, estudios como los de Repetti, Taylor y Seeman (2002) confirman que estilos parentales caracterizados por niveles inadecuados de afecto, de apoyo y predominio de la agresión y el rechazo hacia los hijos se relacionan con la manifestación de problemas conductuales de agresividad, hostilidad y delincuencia. Y trabajos como los de Povedano, Hendry, Ramos y Varela (2011) confirman que la percepción por parte del adolescente de un clima familiar cohesivo, en el que se fomenta el apoyo y la membrecía de los integrantes, con relaciones comunicativas abiertas y positivas, parece amortiguar la situación de distrés o malestar psicológico que supone sufrir de forma continuada la violencia escolar en sus diferentes formas.

Del mismo modo, se consideran como relevantes las intervenciones centradas en el alumnado, y se destaca entre ellas las propuestas de ayuda entre iguales que Cowie y Wallace (2000) clasifican en dos grupos: por un lado, las intervenciones que enfatizan la educación entre iguales, como el aprendizaje cooperativo, siendo este último una metodología útil para reducir las conductas de acoso en el aula (León, Gozalo, \& Polo, 2012) y para adquirir habilidades y competencias sociales (Echeita, 1995; León, Gozalo, \&Vicente, 2004; Eceiza, Arrieta, \& Goñi, 2008; López, Bilbao, \& Rodríguez, 2012) que ayuden a mejorar la interacción en el grupo de iguales; y, por otro lado, las intervenciones que enfatizan el apoyo emocional, concretamente el trabajo de la inteligencia emocional en las aulas (Garaigordobil \& Oñederra, 2010), ya que un déficit en este tipo de inteligencia provoca y facilita la aparición de problemas conductuales en el ámbito de las relaciones interpersonales (León, 2009). Intervenciones todas ellas que podrán paliar los efectos adversos que el acoso escolar tiene tanto para el agresor como pa- 
ra la víctima, alumnos implicados en situaciones de acoso escolar que presentan dificultades de interacción social, siendo necesario restablecer la sensibilidad emocional hacia sí mismos (Sánchez \& Fernández, 2007) y hacia los demás (Avilés \& Monjas, 2005).

\section{Referencias}

Avilés, J. M. (2006). Bullying: el maltrato entre iguales. Agresores, víctimas y testigos en la escuela. Salamanca: Amarú.

Avilés, J. M. (2009). Victimización percibida y bullying: factores diferenciales entre víctimas. Boletín de Psicología, 95, 7-28.

Avilés, J. M., \& Monjas, I. (2005). Estudio de incidencia de la intimidación y el maltrato entre iguales en la educación secundaria obligatoria mediante el cuestionario CIME I -Cuestionario sobre intimidación y maltrato entre iguales-. Anales de Psicología, 21(1), 27-41.

Baldry, A. C., \& Farrington, D. (2005). Protective factors as moderators of risk factors in adolescence bullying. Social Psychology of Education, 8, 263-284.

Benítez, J. L., \& Justicia, F. (2006). El maltrato entre iguales: descripción y análisis del fenómeno. Revista de Investigación Psicoeducativa, 4(2), 81-93.

Berger, C. (2012). Trayectorias de victimización escolar: características y factores de riesgo en adolescentes chilenos. Universitas Psychologica, 11(1), 103-118.

Buelga, S., Cava, M. J., \& Musitu, G. (2012a). Validación de la Escala de Victimización entre Adolescentes a través del Teléfono Móvil y de Internet. Revista Panamericana de Salud, 32(1), 36-42.

Buelga, S., Cava, M. J., \& Musitu, G. (2012b). Reputación social, ajuste psicosocial y victimización entre adolescentes en el contexto escolar. Anales de Psicología, 28(1), 180-187.

Caspi, A., Moffitt, T. E., Silva P. A., Stouthamer-Loeber, M., Krueger, R. F., \& Schmutte, P. S. (1994). Are some people crime-prone? Replications of the personality-crime relationship across countries, genders, races, and methods. Criminology, 32(2), 163-95.

Caurcel, M. J., \& Almeida, A. (2008). La perspectiva moral de las relaciones de victimización entre iguales: un análisis exploratorio de las atribuciones de adolescentes españoles y portugueses. European Journal of Education and Psychology, 1(1), 51-68.

Cava, M. J., Buelga, S., Musitu, G., \& Murgui, S. (2010). Violencia escolar entre adolescentes y sus implicaciones en el ajuste psicosocial: un estudio longitudinal. Revista de Psicodidáctica, 15(1), 21-34.

Cava, M. J., Musitu, G., \& Murgui, S. (2007). Individual and social risk factors related to victimization in a sample of Spanish adolescents. Psychological Reports, 101(1), 275-290.

Cerezo, F. (1997). Conductas agresivas en la edad escolar. Madrid: Pirámide.

Cerezo, F. (2001). Variables de personalidad asociadas a la dinámica bullying (agresores versus víctimas) en niños y niñas de 10 a 15 años. Anales de Psicología, 17(1), 37-44.

Cerezo, F. (junio, 2002). El bullying y su relación con las actitudes de socialización en una muestra de adolescentes. Revista Electrónica Interuniversitaria de Formación del Profesorado, 5(1). Disponible en http://www.aufop.com/aufop/uploaded_files/articulos/1227717262.pdf

Cerezo, F. (2008). Acoso escolar entre iguales. Efectos del bullying. Boletín de Pediatría, 48, 353-368.

Cerezo, F. (2009). Bullying: análisis de la situación en las aulas españolas. International Journal of Psychology and Psychological Therapy, 9(3), 367-378.

Cerezo, F., \& Ato, M. (2005). Bullying among peers in Spanish and English pupils: A sociometric perspective using the BULL-S Questionnaire. Educational Psychology, 25(4), 353-367.

Cowie, H., \& Wallace, P. (2000). Peer support in action. London: Sage.

Defensor del Pueblo (1999). Informe del Defensor del Pueblo sobre violencia escolar. Madrid: Publicaciones del Defensor del Pueblo.

Del Barrio, C., Martin, E., Montero, I., Gutiérrez, H., Barrios, A., \& De Dios, M. J. (2008). Bullying and social exclusion in Spanish secondary school: $\mathrm{Na}$ tional trends from 1999 to 2006. International Journal of Clinical and Health Psychology, 8(3), 657-677.

Díaz-Aguado, M. J. (2005). La violencia entre iguales en la adolescencia y su prevención desde la escuela. Psicothema, 17(4), 549-558. 


\section{María Isabel Polo del Río, Benito León del Barco, Elena Felipe Castaño, Fernando Fajardo Bullón, Teresa Gómez Carroza}

Díaz-Aguado, M. J., Martínez, R., \& Martín, G. (2004). Prevención de la violencia y lucha contra la exclusión desde la adolescencia, 1. La violencia entre iguales en la escuela y en el ocio. Estudios comparativos e instrumentos de evaluación. Madrid: Instituto de la Juventud.

Eceiza, M., Arrieta, M., \& Goñi, A. (2008). Habilidades sociales y contextos de la conducta social. Revista de Psicodidáctica, 13(1), 11-26.

Echeita, G. (1995). El aprendizaje cooperativo. Un análisis psicosocial de sus ventajas respecto a otras estructuras de aprendizaje. En P. Fernández \& A. Melero (Comps.), La interacción social en contextos educativos (pp. 167-189). Madrid: Siglo XXI.

España, Defensor del Pueblo. (2007). Violencia escolar: el maltrato entre iguales en la Educación Secundaria Obligatoria 1999-2006 (Informes, esudios y documentos: 22). Madrid: Publicaciones de la Oficina del Defensor del Pueblo.

Garaigordobil, M., \& Oñederra, J. A. (2010a). Inteligencia emocional en las víctimas de acoso escolar y en los agresores. European Journal of Education and Psychology, 3(2), 243-256.

Garaigordobil, M., \& Oñederra, J. A. (2010b). Un estudio comparativo de las conductas de acoso escolar percibidas por los directivos de los centros educativos y por los estudiantes de 10 a 16 años. En J. J. Gázquez \& M.C. Pérez (Eds.), Investigación en convivencia escolar: variables relacionadas (pp. 97-104). Granada: GEU.

Griffin, R. S., \& Gross, A. M. (2004). Childhood bullying: Current empirical findings and future directions for research. Aggression and Violenta Behavior, 9(4), 379-400.

Hunter, S. C., Mora-Merchán, J. A., \& Ortega, R. (2004). The long-term effects of coping strategy use in the victims of bullying. The Spanish Journal of Psychology, 7(1), 3-12.

Lahire, B. (2007). Infancia y adolescencia: de los tiempos de socialización sometidos a constricciones múltiples. Revista de Antropología Social, 16, 21-38.

León, B. (2009). Salud mental en las aulas. Revista de Estudios de Juventud, 84, 66-83.

León, B., Gozalo, M., \& Polo, I. (2012). Aprendizaje cooperativo y acoso entre iguales. Infancia y Apre- ndizaje: Journal for the Study of Education and Development, 31(1), 23-35.

León, B., Gozalo, M., \& Vicente, F. (2004). Factores interpersonales que condicionan la eficacia del aprendizaje cooperativo. Ciencia Psicológica, 9, 79-92.

López, V., Bilbao, M. A., \& Rodríguez, J. I. (2012). La sala de clases sí importa: incidencia del clima de aula sobre la percepción de intimidación y victimización entre escolares. Universitas Psychologica, 11(1), 91-101.

Martín, E., Fernández, I., Andrés, S., Del Barrio, C., \& Echeita, G. (2003). La intervención para la mejora de la convivencia en los centros educativos: modelos y ámbitos. Infancia y Aprendizaje, 26(1), 79-95.

Martínez-Antón, M., Buelga, S., \& Cava, M. J. (2007). La satisfacción con la vida en la adolescencia y su relación con la autoestima y el ajuste escolar. Anuario de Psicología, 38(2), 293-303.

Martínez-Monteagudo, M. C., Inglés, C. J., Trianes, M. V., \& García-Fernández, J. M. (2011). Perfiles de ansiedad escolar: diferencias en clima social y violencia entre iguales. Electronic Journal of Research in Educational Psychology, 9(25), 1023-1042.

McConville, D. W., \& Cornell, D. G. (2003). Aggressive attitudes predict aggressive behaviour in middle school students. Journal of Emotional and Behavioral Disorders, 11(3), 179-187.

Montañés, M., Bartolomé, R., Parra, M., \& Montañés, J. (2009). El problema del maltrato y el acoso entre iguales en las aulas. Ensayos: Revista de la Facultad de Educación de Albacete, 24, 1-13.

Moreno, M., Vacas, C., \& Roa, J. M. (2006). Víctimización escolar y clima sociofamiliar. Revista Iberoamericana de Educación, 40(6), 12-17.

Musitu, G., \& Cava, M. J. (2003). El rol del apoyo social en el ajuste de los adolescentes. Intervención Psicosocial, 12(2), 179-192.

Musitu, G., Jiménez, T. I., \& Povedano, A. (enero, 2009). Familia y escuela: escenarios de riesgo y protección en la violencia escolar. Revista Electrónica de Motivación y Emoción, 12(32-33).

Olweus, D. (1983). Low school achievement and agressive behaviour in adolescent boys. En D. Magnusson \& V. Allen (Eds.), Human development: An interactional perspective (pp. 353-365). Nueva York: Academic Press. 
Olweus, D. (1993). Bullying at school: What we know and what we can do. Cambridge, MA: Blackwell.

Olweus, D. (1998). Conductas de acoso y amenaza entre escolares. Madrid: Morata.

Olweus, D., \& Endresen, I. M. (1998). The importance of sex-of-stimulus object: Age trends and sex differences in empathic responsiveness. Social Development, 7(3), 370-388.

Oñate, A., \& Piñuel, I. (2005). Informe Cisneros VII: Violencia y acoso escolar en alumnos de Primaria, ESO y Bachiller. Madrid: Instituto de Innovación Educativa y Desarrollo Directivo.

Pellegrini, A., Bartini, M., \& Brooks, F. (1999). School bullies, victims, and aggressive victims: Factors relating to group affiliation and victimization in early adolescence. Journal of Educational Psychology, 91(2), 216-224.

Perren, S., \& Alsaker, F. D. (2006). Social behaviour and peer relationships of victims, bully-victims, and bullies in kindergarten. The Journal of Child Psychology and Psychiatry and Allied Disciplines, 47(1), 45-57.

Povedano, A., Hendry, L., Ramos, M., \& Varela, R. (2011). Victimización escolar: clima familiar, autoestima y satisfacción con la vida desde una perspectiva de género. Psychosocial Intervention, 20(1), 5-12.

Repetti, R. L., Taylor, S. E., \& Seeman, T. E. (2002). Risky families: Family social environments and the mental and physical health of offspring. Psychological Bulletin, 128(2), 330-366.
Rigby, K. (2000). Effects of peer victimisation in schools and perceived social support in adolescent wellbeing. Journal of Adolescence, 23(1), 57-68.

Salmivalli, C., Lagerspetz, K., Björkqvist, K., Österman, K., \& Kaukiainen, A. (1996). Bullying as a group process: Participant roles and their relations to social status within the group. Aggressive Behavior, 22(1), 1-15.

Silva, F., \& Martorell, M. C. (1989). BAS-3, Batería de socialización (Autoinforme). Madrid: TEA.

Sánchez, A., \& Fernández, M. P. (2007). Característica de la agresividad en la adolescencia: diferencias en función del ciclo educativo y del sexo. EduPsykhé, 6(1), 49-83.

Schwartz, D., Dodge, K., Pettit, G., \& Bates, J. (1977). The early socialization of aggressive victims. Child Development, 68(4), 665-675.

Smith, R., Talamelli, L., Cowie, H., Naylor, P., \& Chauhan, P. (2004). Profiles of non-victims, escaped victims, continuing victims and new victims of school bullying. British Journal of Educational Psychology, 74(Pt 4), 565-581.

Sutton, J., \& Smith, P. K. (1999). Social cognition and bullying: Social inadequacy or skilled manipulation? British J. of Developmental Psychology, 17(3), 435-450.

Trianes, M. V., Muñoz, A., \& Jiménez, M. (2007). Las relaciones sociales en la infancia y la adolescencia y sus problemas. Madrid: Pirámide. 
